\title{
Neuroscientific Evidence Against Depression as an Evolutionary Adaptation
}

\author{
Gonzalo Munevar, Donna Irvan \\ Lawrence Technological University, Southfield, USA
}

\begin{abstract}
We will discuss evidence from neuroscience against the hypothesis that depression is cognitively adaptive. A recent version of the notion that depression has adaptive value has been proposed by Andrews and Thomson. Their analytical rumination hypothesis holds that in entering depression an individual is better able to allocate cognitive resources to the very problems that triggered the depressive episode. The result is that depression allows for more analytical and focused thinking about one's most serious personal problems. Depression would thus be adaptive in a way analogous to disease responses such as fever, which gives an advantage to white blood cells over harmful pathogens, thus making it more likely that the individual organism will survive. It is unpleasant but advantageous. Evidence from neuroscience, however, casts doubt on this hypothesis. Some of the key areas involved in the neuroanatomical circuit of depression, such as the PFC (prefrontal cortex), the dorsal ACC (anterior cingulate cortex), and the amygdala-hippocampal path, when adversely affected, lead instead to impaired memory and concentration. Studies have found that depressed patients exhibit cell death and reduced volume in the PFC, decreased activity in the dorsal ACC, and increased volume in the amygdala, while the hippocampus volume is decreased, both of which might be expected to lead to difficulties with memory and problem-solving. Furthermore, some of these areas are critical to the allocation of cognitive resources, thus making the analytical rumination hypothesis even more implausible.
\end{abstract}

Keywords: depression, adaptive, problem-solving, cognitive resources, PFC (prefrontal cortex), anterior cingulate, amygdala, hippocampus

\section{Introduction}

In this paper, we discuss evidence from neuroscience against a new and intriguing hypothesis by Andrews and Thomson (2009) that views depression as a form of cognitive adaptation. As we will see, the claims made in favor of views depression are inconsistent with depression-correlated anatomical and functional deficits in brain regions involved in solving cognitive problems.

Andrews and Thomson (2009) suggest that depression evolved in response to complex problems that require rumination and analytical thinking and is thus evolutionarily adaptive. This idea is referred to as the analytical rumination hypothesis, which holds that the adaptive nature of depression disrupts the normal distribution of cognitive resources between two or more tasks so as to allocate more resources to the solution of

Gonzalo Munevar, Ph.D., professor, Department of Humanities, Social Sciences, and Communication, Lawrence Technological University.

Donna Irvan, B.S., research assistant, Department of Humanities, Social Sciences, and Communication, Lawrence Technological University. 
a critical problem (the problem to which depression is the response). This allows an individual time to concentrate and ruminate over that problem without being distracted by other events or thoughts.

These analytical processes are proposed by Andrews and Thomson (2009) to help generate solutions to critical problems at the expense of other goals. While facing two or more tasks, the more urgent and complex problem will thus monopolize the cognitive resources of the individual. Monopolizing the limited cognitive resources for analytical thinking greatly taxes them, preventing attention to other matters. A result of this biological trade-off is the depressed mood. Since cognitive resources are limited, monopolizing them for any one task will detract resources from other less pressing tasks.

The analytical rumination hypothesis also proposes that the mind is allowed to become more focused and analytical by creating a lack of interest, or anhedonia, in many otherwise pleasurable experiences (Andrews \& Thomson, 2009). Many complex problems do normally require concentration and time away from other distractions, although usually not to the extent of reducing pleasure from normal social and rewarding experiences. The significance of this lack of interest, according to this hypothesis, is that the mind no longer seeks activities that draw attention away from one especially significant problem. Creating a lack of interest and pleasure in other activities, such as social events, hobbies, sexual relations, and even normal eating and sleep patterns, is thought to further isolate one from distractions, thereby promoting uninterrupted rumination (Andrews \& Thomson, 2009). The individual can now be solely devoted to problem-solving and focused thinking because the saliency of other tasks and interests is suppressed. In summary, then, Andrews and Thomson (2009) suggest that depression promotes productive analytical processes to the detriment of other mental activities, helping individuals come up with solutions to the problems that may have originally created their depressive state.

Initially, the analytical rumination hypothesis may sound attractive and plausible. Instead of depression being maladaptive and unproductive, now it seems to be beneficial and serves a greater purpose. This way of thinking could greatly reduce the stigma often associated with depression, which sometimes extends to the methods of treatment. This initial plausibility is enhanced by analogy to other physiological conditions and behaviors that have been proven to be evolutionarily adaptive and beneficial even though their action causes discomfort to the individual.

\section{Adaptive Mechanisms}

Fever, for example, is a well-known physiological response that causes inconvenience and discomfort to an individual, yet it serves an adaptive and beneficial function. When pathogens invade the body, the hypothalamus triggers processes that lead to a rise in body temperature. Normal human body temperature is an optimal environment for microbial organisms to thrive and reproduce very quickly. However, when a rise in body temperature occurs due to fever, that optimal environment is severely degraded, greatly reducing the reproductive rate of the pathogen. The drastic decline in pathogen reproduction and proliferation allows the body time to initiate an effective immune response. Moreover, the elevated body temperature makes phagocytes more effective and increases the division of white blood cells, which are then able to engulf and destroy the pathogenic invaders more efficiently (Maier \& Watkins, 1998). The beneficial immunological response comes at a great metabolic cost however, often leaving an individual feeling uncomfortable and weak for a period of time. The biological trade-off for such an important immune response is the episode of weakness while the body tries to regain a point of homeostasis. The proposed adaptive depression described above-a 
period of depression and discomfort while the mind ruminates and analytically tries to resolve complex problems - seems analogous to this uncontroversial adaptive physiological mechanism.

Unlike the beneficial adaptive nature of fever, however, the proposed analytical rumination hypothesis does not stand up to the scrutiny of neurobiological evidence. The main objection to the hypothesis is that three key areas of the brain are adversely affected by episodes of depression, and that malfunction or disruptions in any of these three key areas are known to lead to impaired concentration and memory, not improved cognition. These three key areas in the brain are the PFC (prefrontal cortex), ACC (anterior cingulate cortex), and the hippocampal-amygdala region. Additionally, these three areas often interact with one another, so that if one of them is not functioning properly, it can cause disruption in the other areas as well, further reducing cognitive function.

\section{PFC}

First, based on data accumulated from neuroimaging and postmortem studies, the PFC seems to be highly involved with major depression (Liotti \& Mayberg, 2001). The PFC is an area of the brain commonly associated with the neuropathology of major depression (Rajikowska, 2000). Both cellular atrophy and cellular loss have been found in this area during postmortem study, which is highly significant because the PFC is associated with higher cognitive function. Additionally, the PFC gathers and then integrates incoming information from many different areas of the brain to arrive at solutions to pressing issues. This area is described as "the crucial convergence zone", due in large part to its importance for maintaining attention to the external environment while addressing information regarding internal states such as mood (Liotti \& Mayberg, 2001). This area is also important for executive function and planning, especially in tasks requiring an integration of multiple pieces of information acquired over time (Gazzaniga, Ivry, \& Mangun, 2009, p. 73). Cellular loss and atrophy in such an important structure does not seem conducive to focused thought processes. Analytical processing and rumination imply an ability to break apart and scrutinize various bits of information to help devise a resolution strategy. Since the PFC is important for planning and integrating information, damage or impaired function of this important brain structure would place any animal at a disadvantage. But that is precisely what happens during depression (Rajikowska, 2000).

\section{ACC}

Second, the ACC is vital for relaying information from the limbic area of the brain to the PFC (Liotti \& Mayberg, 2001). The limbic system is an area well known for processing emotions, so it seems logical that any impairment or dysfunction of the ACC would be relevant to depressive emotional states. Disruption of the delivery of emotionally significant information to the PFC would naturally affect mood, perhaps causing an emotional detachment from, or indifference towards the very problem that needs resolution. Additionally, the ACC is crucial for attentional processing and, according to Liotti and Mayberg (2001), decreased activity in the dorsal region of the ACC is involved with depression.

Moreover, evidence is accumulating suggesting that the ACC is a critical component of an important monitoring system that becomes more active during divided-attention tasks requiring a high level of attention (Gazzaniga, Ivry, \& Mangun, 2009, p. 589). The need for a system that monitors and coordinates activation of certain areas of the brain under changing circumstances seems obvious. As the external environment is ever changing, the incoming information involved with initiating an effective and appropriate response must be 
updated and evaluated continuously. As the level of difficulty or novelty of conditions change, blood flow in the cingulate area also changes, which implies that this area is involved in attentional tasks. One important factor relevant in a monitoring system would be error detection. Error detection is necessary to ensure that an ineffective or incorrect response is not repeated. Using evoked-potential studies, a correlation has been found between the occurrence of committing an error and an electrophysiological signal that can be detected in the PFC. More specifically, this electrophysiological signal, referred to as the ERN (error-related negativity) response, has been localized to the ACC (Dehaene, Posner, \& Tucker, 1994).

A slightly differing view is offered by Cohen, Botvinick, and Carter (2000), which suggests that, instead of just error detection, the ACC is involved with response conflict. Their analysis is based on a study by Gehring and Knight (2000) that used a variant design of the Stroop task to manipulate two factors of interest: degree of difficulty and congruency of color-word cues. ACC activation appears to be sensitive to the degree of the response conflict presented; different color-word cues prompt greater ACC activation (Gehring \& Knight, 2000). This view implies that the ACC is not just involved with error detection, but more specifically when there is a conflict involved. It is possible that when the ACC responds to conflict, two different but parallel routes of control can be activated. One route would increase selective attention and the other route would prepare for some form of response (Cohen, Botvinick, \& Carter, 2000). In addition, extensive work done by Gehring and Knight (2000) has shown that the PFC may supply information to the ACC that is needed for determining between correct and incorrect responses. In line with this view, the ACC would monitor for errors and conflict and then other areas of the brain would carry out the appropriate action.

Profound and complex problems arising in life are bound to be rife with conflict, so the analytical rumination hypothesis suggests that depression may offer a window of opportunity to analyze and resolve these conflicts. However, it seems counterintuitive that decreased activity in an area of the brain that processes attention would facilitate the resolution of any problems. Decreases in activity of the ACC, as is often seen in depression, would seem to diminish rather than enhance concentration, focus, and cognitive strategies.

\section{Hippocampus and Amygdala}

Lastly, the hippocampus and the amygdala are brain structures highly involved with memory and emotion. Several studies suggest that there is a high probability that the amygdala modulates the hippocampal memory consolidation of emotionally arousing episodes. The interaction between the role of the amygdala in emotion and the memory function of the hippocampus is thought to ensure that certain emotionally charged events will be remembered. This is important for avoiding a dangerous or unpleasant experience or for repeating a rewarding and enjoyable event in the future (Gazzaniga et al., 2009, pp. 377-378). The amygdala is necessary for implicit emotional learning and interacts with the hippocampal memory system regarding emotional information and events. Appropriate emotional responses to external stimuli laden with emotional relevance rely on a functioning amygdala (Gazzaniga et al., 2009, p. 374).

Impaired arousal-mediated memory consolidation was found in a population of patients that had suffered damage to the medial temporal lobe (which includes the amygdala), providing substantiation that this structure most likely participates in the formation of emotional memory. The amygdala and hippocampus cooperatively regulate the consolidation of declarative memory for arousing events and give an understanding as to how brain regions that coordinate emotional and cognitive functions drive mental activity (LaBar \& Phelps, 1998). Most complex problems that require deep thought and rumination surely would have many emotional nuances that 
would involve the hippocampus and the amygdala. Interestingly, and in contrast to what the analytical rumination hypothesis implies, Kleinsmith and Kaplan (1963) found that during low-arousal states, there is a significantly lessened retention of learned material. The low-arousal state that would coincide with depression in the form of isolation from other activities and being more sedate would lessen the chance that innovative resolutions would be reached. On the other hand, with greater arousal, there is a poorer immediate result, but the retention is greater, hence we achieve better learning in conditions of high arousal (Kleinsmith \& Kaplan, 1963).

A problem for the analytical rumination hypothesis is the finding that there is a reduced volume in the hippocampus and an increased volume in the amygdala in individuals experiencing depression (Bremner, Narayan, Anderson, Staib, Miller, \& Charney, 2000). This finding is relevant because an enlarged amygdala may trigger an overly exaggerated emphasis on negative emotional memories, which seems likely to induce more brooding than problem resolution. The decreased volume in the hippocampus implies some memory impairments, which could interfere with memory and thus with the ability to concentrate appropriately on critical problems.

Andrews and Thomson (2009) agree that decreases in hippocampal volume are often seen in depression; however, they point out that the reduction in volume is not solely restricted to depression. Volume reduction is also seen in individuals suffering from chronic stress (Andrews \& Thomson, 2009). Indeed there are a variety of disorders where a reduction in the volume of the hippocampus is apparent. Nevertheless, the shared decrease in hippocampal reduction found both in depression and other disorders, or even just as a response to chronic stress, does not detract from the fact that these decreases usually impair cognitive abilities. Hence, the fact that decreases in volume of the hippocampus is not limited to depression does not provide support for the analytical rumination hypothesis. If anything, it reinforces the idea that depressed individuals will suffer cognitive deficits due to hippocampal damage. These cognitive deficits are likely to make finding solutions to pressing complex problems even more difficult.

\section{Conclusions}

Based on the evidence from neuroscience, depression does not appear to be conducive to useful rumination and analytical thinking. One only needs to consider the areas in the brain that are affected by depression to infer that depression would not be evolutionary adaptive or beneficial to cognitive processes. If areas of the brain that enable and enhance cognitive function are impaired, it only stands to reason that being able to analyze complex problems would be less likely. It seems more likely that a reduction in concentration, disrupted information relays, and impaired memory, as is often seen in depressed individuals, would exacerbate any pressing complex problems, rather than facilitate resolution. Though the analytical rumination hypothesis sounds attractive on the surface, depression does not appear to be a cognitively adaptive function.

The very core of the analytical rumination hypothesis by Andrews and Thomson (2009) seems to fall apart from a careful consideration of the evidence. Cohen et al. (2000) tried to determine the structures "that are involved in monitoring performance and deciding how and when to allocate control - that is determining when a goal is not being satisfactorily met and deciding to devote more effort to it” (p. 421). Based on Gehring and Knight's study (2000), Cohen et al. (2000) concluded that those structures are precisely the PFC and the ACC, two of the structures most adversely affected in depression.

Solving complex and analytical problems does indeed require concentration, selective attention, and 
rumination time as is suggested by the analytical rumination hypothesis; however, a depressed state of mind and its consequences are most likely not the optimal physical nor mental environment from which to make these pivotal and important decisions. More beneficial would be a brain that has all of these key areas involved with planning, execution, and reasoning to be working at their very best.

\section{References}

Andrews, P. W., \& Thomson, Jr., J. A. (2009). The bright side of being blue: Depression as an adaptation for analyzing complex problems. Psychological Review, 116(3), 1-69.

Bremner, J. D., Narayan, M., Anderson, E. R., Staib, L. H., Miller, H. L., \& Charney, D. S. (2000). Hippocampal volume reduction in major depression. American Journal of Psychiatry, 157, 115-118.

Cohen, J. D., Botvinick, M., \& Carter, C. S. (2000). Anterior cingulate and prefrontal cortex: Who's in control? Nature Neuroscience, 3(5), 421-423.

Dehaene, S., Posner, M. I., \& Tucker, D. M. (1994). Localization of a neural system for error detection and compensation. Psychological Science, 5, 303-305.

Gazzaniga, M. S., Ivry, R. B., \& Mangun, G. R. (2009). Cognitive Neuroscience: The biology of the mind (3rd ed.). New York, N. Y.: W. W. Norton \& Company.

Gehring, W. J., \& Knight, R. T. (2000). Prefrontal-cingulate interactions in action monitorning. Nature Neuroscience, 5, 516-520.

Kleinsmith, L. J., \& Kaplan, S. (1963). Paired-associate learning as a function of arousal and interpolated interval. Journal of Experimental Psychology, 65(2), 190-193.

LaBar, K. S., \& Phelps, E. A. (1998). Arousal-mediated memory consolidation: Role of the medial temporal lobe in humans. Psychological Science, 9(6), 490-493.

Liotti, M., \& Mayberg, H. S. (2001). The role of functional neuroimaging in the neuropsychology of depression. Journal of Clinical and Experimental Neuropsychology, 23, 121-136.

Maier, S. F., \& Watkins, L. R. (1998). Cytokines for psychologists: Implications of bidirectional immune-to-brain communication for understanding behavior, mood, and cognition. Psychological Review, 105(1), 83-107.

Rajikowska, G. (2000). Postmortem studies in mood disorders indicate altered numbers of neurons and glial cells. Biological Psychiatry, 48, 766-777. 